\title{
Computer-Aided Design for Dimensional Stability of Injection Molded Fiber- Reinforced Polymers
}

\section{Mr. Christopher J. Hershey, Michigan State University}

Christopher Hershey is a third year Chemical Engineering doctoral student and a Graduate Research Assistant in the Department of Chemical Engineering and Materials Science at Michigan State University. He received dual BS degrees in Chemical Engineering and Computational Chemistry at Michigan State University. During his undergraduate studies, he worked as a Resident Assistant for four years as well as an Undergraduate Research Assistant focusing on computational fluid dynamics simulations of reactive polyurethane foam flow. His current research interests include the nonlinear rheology and constitutive modeling of polymer nanocomposites.

\section{Dr. K. (Jay) Jayaraman, Michigan State University}

Dr. K. (Jay) Jayaraman is Professor and Withrow Distinguished Scholar in the Department of Chemical Engineering and Materials Science at Michigan State University. He earned a PhD degree in chemical engineering from Princeton University. His research interests are in rheological characterization, melt processing and solid-state processing of polymer composites and nanocomposites, foams and thermoplastic elastomers. He has collaborated continually with companies such as ExxonMobil Chemical Co., Dow Chemical Co., Basell, Siemens, BASF, Petoskey Plastics, Nanocor and Eovations LLC on applying rheological tests to understand flow behavior of polymeric materials: flow marks in injection molding, processing and rheology of polymer-clay nanocomposites, foamed polymers, and solid state die-drawing of expanded and oriented polymer composites. He has supervised $23 \mathrm{PhD}$ students and $18 \mathrm{MS}$ students at Michigan State University. He has co-authored over seventy publications, five patents and one filed patent and delivered keynote lectures at meetings of the International Polymer Processing Society. 


\title{
Computer-aided design for dimensional stability of injection molded fiber-reinforced polymers
}

\begin{abstract}
New exercises were developed for a senior level composites processing class with the help of Moldex3D software "e-design" modules to illustrate computer aided process design for injection molding of short fiber reinforced polypropylene parts. This class combines laboratory measurements and lectures on problem solving related to extrusion, compression molding and injection molding of both thermoplastic and thermosetting compounds. The emphasis in these problems was on dimensional stability and fiber orientation with different part geometries - a center gated disc and an edge gated rectangular plaque. Sixty students enrolled in the class were grouped into teams of three for this problem set.
\end{abstract}

The geometry and mesh were generated and made available to the student teams. Two tutorials were also run to demonstrate the use of the software with readily available meshes to the students. By changing different parameters such as fill time, pack time, polymer type and fiber content, students could visualize these changes in the molded part dimensions and shape. Part quality was then estimated using parameters such as overall shrinkage and warpage. Students were also able to directly compare the simulated disc to an actual injection molded disc from a similar mold. The advantage of the simulation exercise was in helping students to visualize flow fields during injection as well as understanding the effects of various parameters on the final part dimensions. This visualization was a strength of Moldex3D as students were able to generate detailed contour plots and graphs along with videos to fully understand the processing stage for polymer composites. Using these visualization tools, each student team was able to evaluate and summarize how incorporating glass fibers into a polymer matrix affected the shrinkage and warpage of the molded part. Student responses were gathered to a survey questionnaire on their learning experience with the computer-aided design exercises.

Introduction

The Composite Materials Processing course presented here is a senior level undergraduate technical elective offered in the chemical engineering curriculum. Students are required to take either this course or the Biochemical Engineering course in order to receive their degree. Over the last few years, there has been an increase in the number of seniors taking this class over the Biochemical Engineering course with nearly seventy students in the latest semester. In the past decade, undergraduate polymer processing courses have emerged across multiple engineering disciplines worldwide. ${ }^{1-3}$ Within these courses, very little development in promoting computer simulations have been discussed. Moreover, this work shows that students can benefit by combining computational tools with hands-on laboratory exercises and that existing courses can readily implement the strategies utilized in the Composite Materials Processing course.

The class consists of two 1-hour lectures and one 3-hour lab per week for a semester. The laboratory offers students a hands-on approach to understanding fundamental polymer processing procedures. In the lab, students are exposed to industrial process such as compression 
molding, extrusion and injection molding of fiber-filled and fiber-free polymers. It is the intent of these labs to show not only how these machines are operated, but to develop the students understanding in the overall product design and quality of a molded part.

In injection molding, molten polymer is pressurized and forced into a cooled mold of a desired shape. In this work, students were asked to mold circular discs using fiber-free and fiber-filled polypropylene. In both of these materials, a certain amount of the final molded product may undergo shrinkage and warpage, reducing the overall quality of the part. Students were asked to characterize these deformations and improve upon them by altering process conditions such as the injection fill time. However, computer simulations were introduced to give students more freedom in modifying process conditions and exploring their effects on the molded part.

Two computational tools were provided to students to enhance their laboratory experience. An injection molding simulation software package, Moldex3D, was coupled with the laboratory exercise giving students a computational tool which replicated the molded circular disc made experimentally. In addition, material models provided by the software were exact matches to the lab polymer in both grade and manufacturer. Students were then asked to perform several simulations on fiber-filled and fiber-free polypropylene ${ }^{4}$ models and determine how the product shrinkage and warpage compared experimentally. A separate home-written program, Sticks, was also used to describe the effect of fiber addition in molded parts. This software gave students a simple resource to learn the fundamentals of percolation due to increased fiber addition.

Both programs, although varying in their degrees of complexity, provided students with an alternative resource to understand the fundamentals of polymer composite processing.

\section{Laboratory Experiment}

Injection molding is one of the most widely used method for processing polymers. It makes sense then that a polymer processing should not only teach this method, but provide a hands-on demonstration of its use in a laboratory exercise.

The experiment of interest in this work required students to operate a Morgan-Press injection molding machine priced around $\$ 25,000$. In this experiment students produced molded circular discs with fiber filled and unfilled polypropylene produced by RTP materials. The RTP polypropylene pellets prices vary on fiber filler content and is available by contacting the manufacturer. The Morgan-Press and molded discs are shown in Figure 1. 
A.

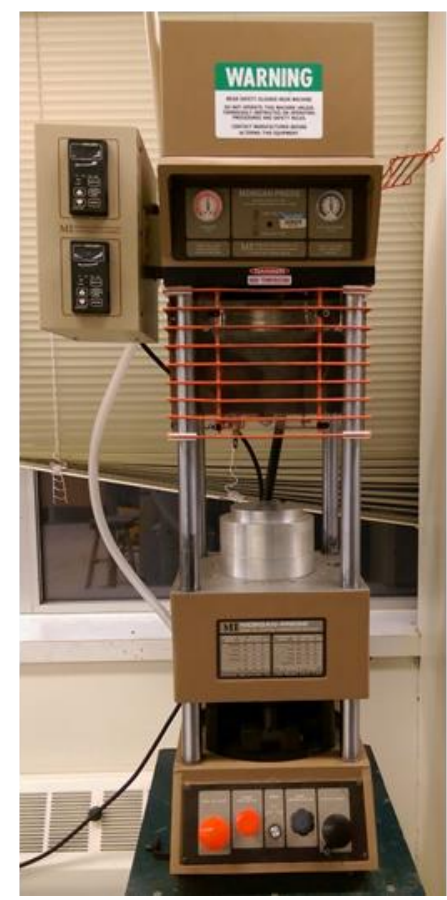

B.

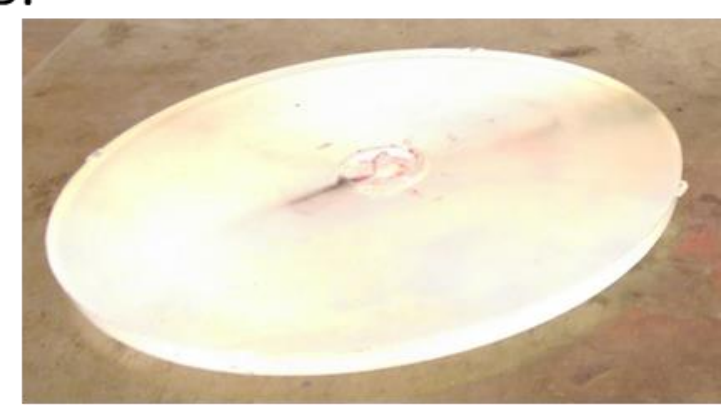

C.

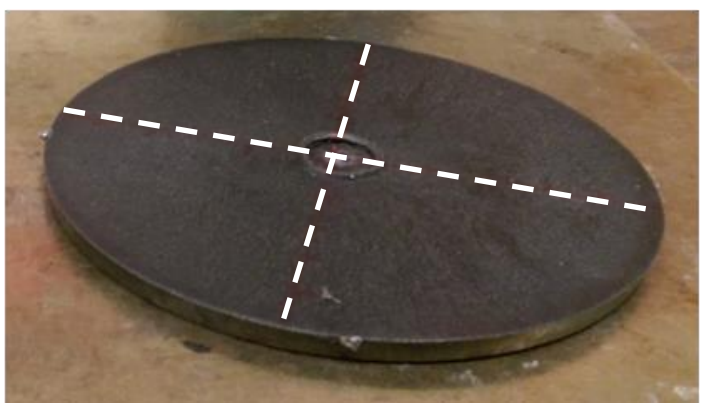

Figure 1. Experimental equipment and molded parts in composites lab: A) Morgan-Press injection molding machine B) Fiber-free polypropylene disc C) Fiber-filled polypropylene disc where dashed lines correspond to warpage measurements.

The objective of this experiment was to have students identify the processing conditions in injection molding which lead to part shrinkage and warpage. Shrinkage is considered the difference in the diameter of the molded part relative to the diameter of the mold itself. Warpage may be considered the degree of bowing in the part in which students measured the thickness of the part along the dashed lines shown in Figure 1C. By measuring the degree of shrinkage and warpage with calipers, students were expected to understand that higher injection pressure and lower pressure gradients lead to less shrinkage and warpage, respectively. After the trends in product quality were successfully identified, students were then asked to repeat the experiment with fiber filled polymer. Students were asked to measure the part using the same procedure as the unfilled polymer case and compare the two samples. This should have demonstrated that the addition of fiber increases the part warpage. Although students were able to measure how the shrinkage and warpage changed with a fiber filled polymer, only with the help of computer simulations were advanced concepts such as fiber orientation properly visualized.

\section{Simulation Software}

The Moldex3D software is classified as a computer aided engineering (CAE) injection molding simulation tool. In the chemical engineering curriculum, students are exposed to a variety of software packages, however this was the first exposure students had to a CAE simulation package. Therefore, it was a requirement that the software be easy to learn. Two lecture periods were all that was required for students to develop a working knowledge of this software to begin their project. The workflow of the two workshops is shown in Figure 2. 


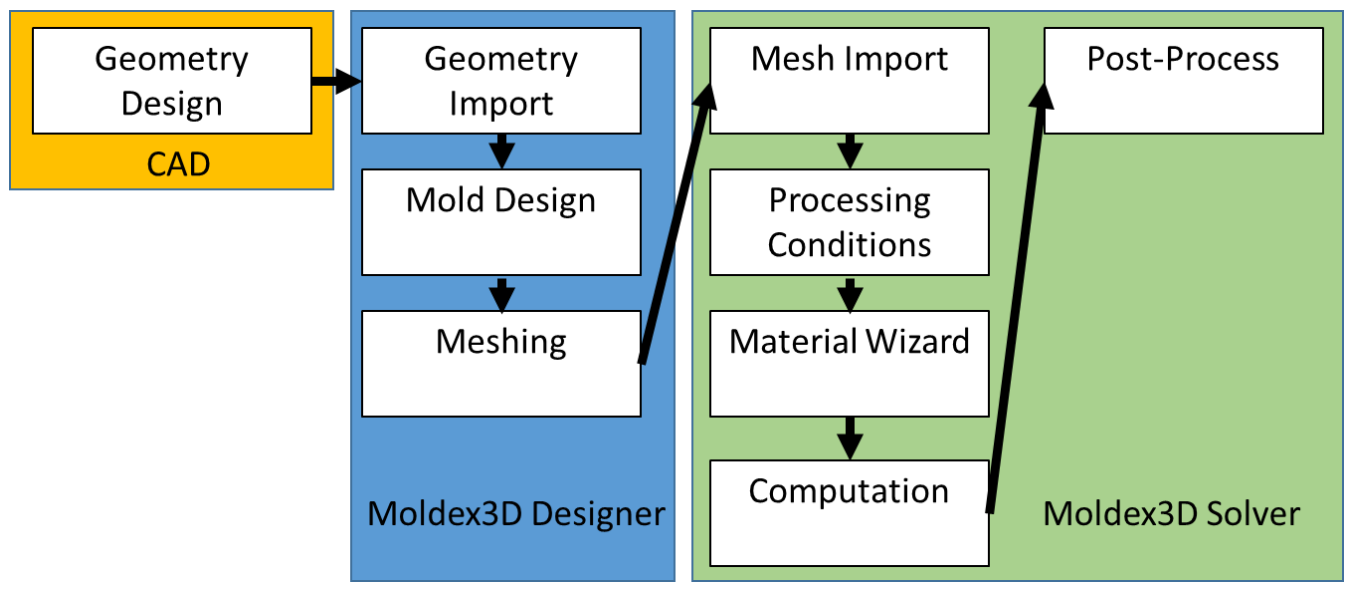

Figure 2. Workflow diagram used in workshop. CAD shown for illustrative purposes with actual geometries and meshes provided to students for simulation.

The software itself is broken up into two parts: Moldex3D Designer and Moldex3D solver. The Moldex3D Designer is used to define injection inlets, cooling channels and to mesh the final geometry. The Moldex3D solver is the main program designed to take user inputs such as processing conditions and material models to simulate the entire injection molding process. There were a total of 70 licenses installed, giving each student the ability to learn these tools independently.

Two geometries, as shown in Figure 3., were meshed and provided to students using a separate computer aided design (CAD) software suite. It should be noted at this point that the circular disc in Figure 3. is of identical dimensions to the discs developed in the laboratory experiment and shown in Figure 2. The design of these geometries are similar to those used by other researchers 5 .
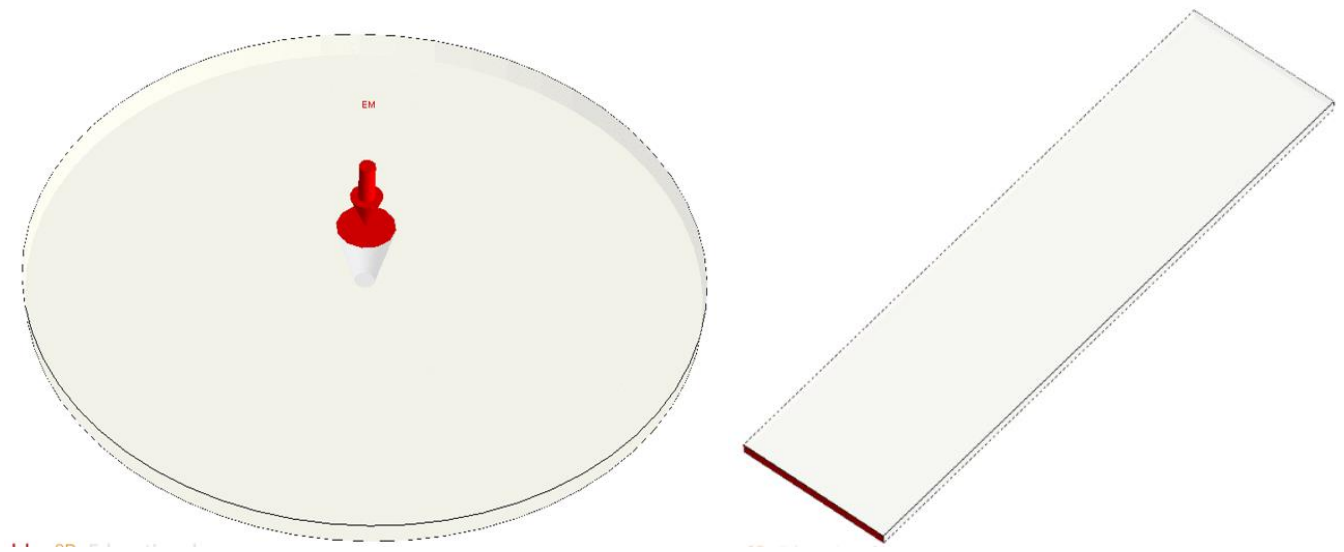

Figure 3. Molded geometries provided to students for workshop: left) center-gated circular disc right) edge-gated rectangular bar.

The Material Wizard included in the software is a detailed compendium of thermosets and thermoplastics with accurate and verified material properties of actual industrial grade polymers. 
Among these polymers were the actual fiber-filled and fiber-free polypropylene used by the students in the laboratory. These materials were used by the students to give the best comparison with their experimental findings.

Solution results generated by the Moldex3D solver included the filling, packing and warpage stages of injection molding. Of particular interest was the warpage calculation, giving students a basis to compare simulated part quality with observed part quality in their molded discs.

In addition to the Moldex3D software, students were also introduced to a home-written program known as Sticks to calculate the percolation threshold of the fiber filler in polymer systems. When fibers are added into polymers, depending on their size and concentration, interactions between these fibers may occur forming a filler network. The Sticks program gives users a set of parameters to vary such as the number of fibers, fiber length, angle and so on. A calculation is then made to determine, in a given lattice, where a network forms as well as its size. The Sticks program is made freely available and may be provided by the authors on request. This simple to use program was not discussed during the workshops, but coupling this software with Moldex3D gives a complete picture of the fiber-filled effect in molded parts.

Implementation

A total of sixty students were enrolled in the Composite Materials Processing course during the scope of this work. Two fifty minute workshops were held to introduce the Moldex3D software suite with the intention of following up with a problem set. This problem set was designed to mimic the laboratory experiment in order to make detailed comparisons.

During the first workshop, students were introduced to using the Moldex3D Designer package. The objective of this first workshop was an introduction to the user interface of the software. After students were acclimated to the interface of the software, a brief overview of the Designer package was given. For assigned project, students were provided the meshed geometries shown in Figure 3. This saved on time and ensured that each student had the same model dimensions and mesh accuracy.

The second workshop utilized the rectangular bar mesh to illustrate the Moldex3D solver package. This workshop needed to teach students in a short duration how to set up a case file by defining realistic processing conditions, choosing a material and its properties and then how to post-process the simulated solution. Since the simulation took nearly 30 minutes to complete, pre-calculated simulations were provided for the post-processing stage. To make a direct comparison with the experimental findings from the lab, students were shown how to export their data from the simulation into representative figures illustrating shrinkage and warpage.

\section{Outcomes Assessment}

Students were given an independent project with the goal of investigating the effect of varying:

- Processing conditions: fill time, pack time and mold temperature

- Mold geometry: circular gated disc and a thin rectangular bar

- Material type: thermosets and thermoplastics 
- Fiber weight fraction

Students were also able to make a direct comparison with the accompanying laboratory experiment by choosing the same material and processing conditions found in the lab. Once the project was completed, students were asked to generate key figures which represented the overall shrinkage and warpage of the simulated parts. These parts included both the circular disc and rectangular bar molded with fiber-filled and fiber-free polymers. Each student was then asked to provide a brief explanation as to what was observed in each simulation and why the observed trends were so.

Students were assessed on how well they understood the results they received from the Moldex3D software as well as the quality of their generated figures. An example of a desired solution to the warpage calculation is given in Figures 4.

Students received full credit if they were successful in making comparisons on how varying the geometry as well as the injection time affected the shrinkage and warpage of the part as well as explain why. The warpage results shown in Figure $4(\mathrm{a}, \mathrm{b})$. compare the geometry effect at different fill times. Students were able to see where the greatest displacement occurred in both geometries and how factors such as the injection inlet affected the pressure gradient and therefore the warpage. In Figure 4c. the effect of fiber addition for a rectangular geometry clearly showed students that fibers increase the non-uniformity in displacement and is therefore a major contributor in part warpage.

The next tier of students that missed points in this project were those that either generated poorly legible plots or incorrectly stated the reasoning to increases in predicted warpage and shrinkage.

Finally, the lower tier of students missed several variations that were asked for with regards to either fill time, fiber addition or geometry effects. The overall average for this project was an $85 \%$ showing that for the most part, students responded well to the project and were able to adequately learn the software in a short amount of time. In addition to understanding the software, students gained a deeper perspective to their experimental work by making direct numerical comparisons to a project they already had a vested interest in. 

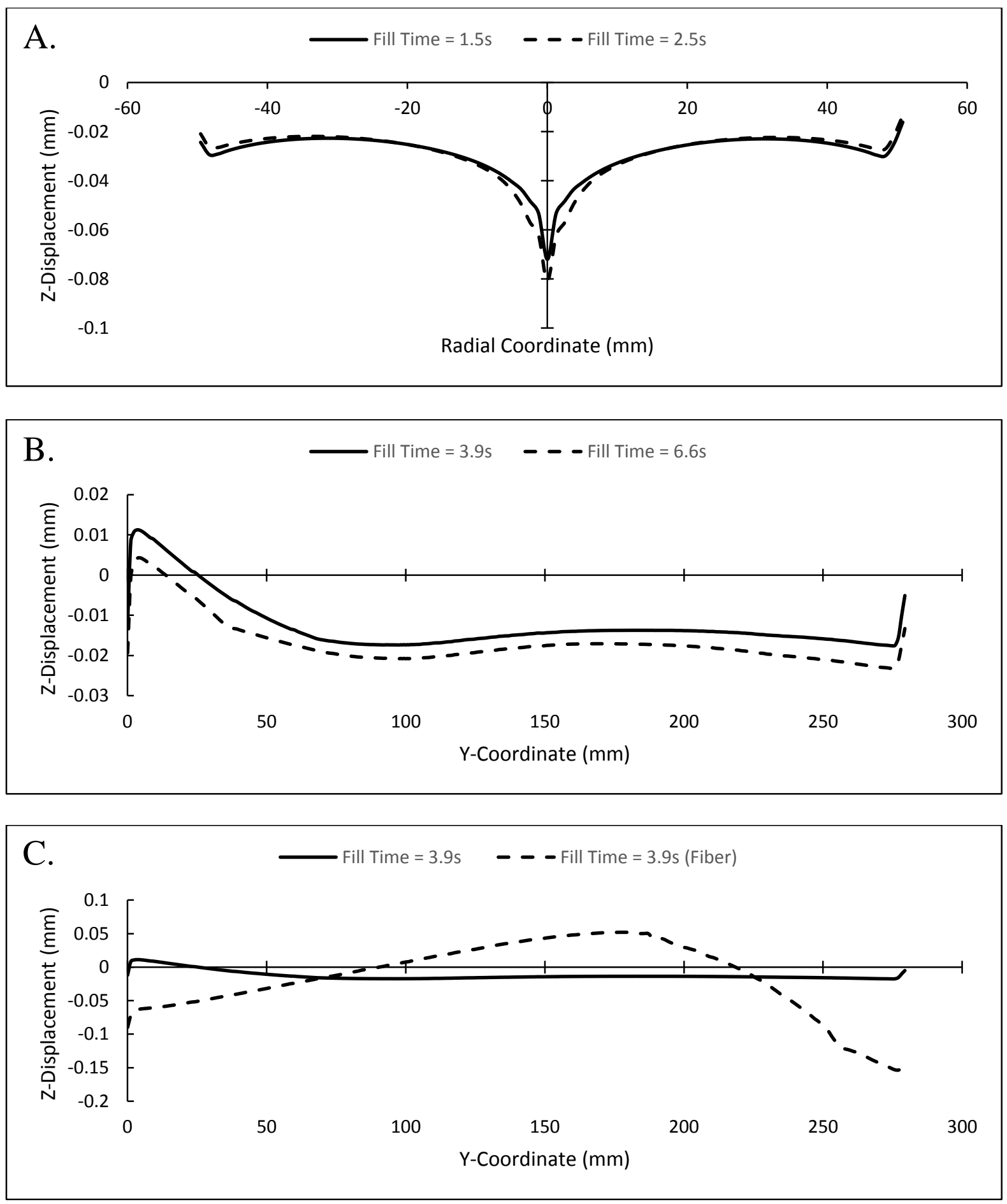

Figure 4. Typical results from injection molding simulations comparing warpage for: (a) circular geometry (b) rectangular geometry (c) fiber filled rectangular geometry

At the end of the project, students were asked several questions regarding their overall experience of the workshop. Some comments included that students enjoyed working with simulation software and gaining a deeper understanding of injection molding. Conversely, there 
were students that thought the simulations took too long to run and that the time commitment did not justify only one assigned project. However, some students suggested that a single geometry should be used in future projects while splitting the project up into smaller parts. In future projects, the simulation of just a circular disc may prove to be the most useful for relating the simulation to the experimental findings in the lab.

\section{Conclusion}

Students enrolled in the senior level Composite Materials Processing course were introduced to a suite of computational tools to complement their laboratory experiments. Each student worked in groups of three during the injection molding exercise given in the lab. During this experiment, each group was asked to investigate the effect that fiber-filled and fiber-free polymers had in the product quality of a circular disc. Product quality was determined by the overall shrinkage and warpage of the molded part. To accompany the laboratory experiments, a two-lecture workshop was developed for the class to teach the injection molding simulation suite, Moldex3D. Students were given enough information during the workshop to complete an assigned project to be worked on independently. A meshed geometry identical to the molded circular disc in the laboratory and a meshed rectangular bar were provided to the students for the project. Each student was asked to vary the processing conditions such as fill time and pressure as well as the type of polymer used: fiber-filled and fiber-free polypropylene. The simulation used an identical geometry and material as used in the lab exercise, thus students had the unique opportunity to make direct comparisons between simulation and experiment. Each student generated a report describing the effect that each variation had on the overall product quality. Given that students had molded only circular discs in the lab, it is clear that the incorporation of computational tools allows for not only direct comparisons to experiments, but broader generalizations of observable trends.

Acknowledgments

The authors would like to thank the staff at Moldex3D for providing the training, software and technical support needed to make this work possible.

\section{References}

1. M. Hawley, K. Jayaraman, J. Sticklen, B. Moore, L. Drzal and R. L. McCullough, Composites Part A, 32, 1175 (2001).

2. A. Mirmiran, L. C. Bank, K. W. Neale, J. T. Mottram, T. Ueda and J. F. Davalos, J. Prof. Issues Eng. Educ. Pract., 129, 155 (2003).

3. W. Hall and S. Palmer, J. Mat. Educ., 37, 155 (2015).

4. Polymer Properties Test Report (Polypropylene, sample report), C-Mold/AC Technology Polymer Laboratories, (1995).

5. P. H. Foss, H. C. Tseng, J. Snawerdt, Y. J. Change, W. H. Yang and C. H. Hsu, Polym. Compos., 35,671 (2014). 